\title{
Pulmonary arteriovenous malformation
}

INSERM

\section{Source}

INSERM. (1999). Orphanet: an online rare disease and orphan drug data base. Pulmonary arteriovenous malformation. ORPHA:2038

Pulmonary arteriovenous malformation (PAVM) describes an anatomic communication between a pulmonary artery and a pulmonary vein leading to a right to left extracardiac shunt that can be asymptomatic or can lead to varying manifestations such as dyspnea, hemoptysis, and neurological symptoms. 